Dhaka Univ. J. Sci. 65(1): 1-7, 2017 (January)

\title{
A Comparative Study on Two Person Zero Sum Game Problems Through Computer Algebra
}

\author{
H. K. Das \\ Department of Mathematics, Dhaka University, Dhaka-1000, Bangladesh
}

(Received: 1 October 2015; Accepted: 28 August 2016)

\begin{abstract}
This paper improves a game theoretic algorithm and develops its computer oriented program using MATHEMATICA for solving two person zero sum game problems. The algorithm and computer algebra are drawn upon mainly from two sources, namely the papers H. K. Das, Saha and Hasan ; H. K. Das and Hasan ${ }^{6}$ being able to solve two person zero sum game problems with single payoff elements. We do a comparative study of the current algorithm and computer technique with the papers ${ }^{5,6}$. We show that the current technique is better than the papers $^{5,6}$ in saving labor and time for solving two person game problems by analyzing a number of numerical examples.
\end{abstract}

Keywords: Game Theory, Pure and Mixed strategy, Dominance, Linear Programming, Computer Algebra.

\section{Introduction}

The individual most closely associated with the creation of the theory of games is John Von Neumann, one of the greatest mathematicians of the 20th century. Although others preceded him in formulating a theory of games - notably Emile Borel - it was von Neumann who published in 1928 the paper that laid the foundation for the theory of twoperson zero-sum games. Von Neumann's work culminated in a fundamental book ${ }^{13}$ on game theory. Other discussions of the theory of games relevant for our present purposes may be found in the text book ${ }^{14,15}$ and papers ${ }^{5,6}$.

The concepts of game theory provide a language to formulate structure, analyze, and understand strategic scenarios. The key fact seems to be that whenever one player's strategy is predictable, the opponent can take great advantage of this information to improve his position. Therefore, an essential feature of a rational plan for playing a game such as this one is that neither player should be able to deduce which strategy the other will use. Hence, in this case, rather than applying some known criterion for determining a single strategy that will definitely be used, it is necessary to choose among alternatives acceptable strategies on some kind of random basis. By doing this, neither player knows in advance which of his own strategies will be used, let alone what his opponent will do.

This suggests, in very general terms, the kind of approach that is required for games lacking for the manual procedure. So far several authors namely Bansal ${ }^{1}$, Brown $^{2}$, Meyerson ${ }^{11}$, Davis $^{4}$, Nisan $^{12}$, Mayer ${ }^{10}$, Hofbauer ${ }^{8}$, H.K. Das ${ }^{5,6,16,17}$ Karak $^{9}$ and many other authors proposed different types of theoretical discussion of game problems with their strategies. But Das ${ }^{5,6}$ discuss computational procedure of game theory. Most of the authors did not discuss the whole problem comprehensively. Hence if we collect data as field work including launching, advertisement, campaigns for competing products and planning war strategies for opposing armies. These situations are in contrast with the ones we have studied so far where nature is viewed a no malevolent opponent. If one wants to win then he must to solve these data to find their strategies and game value. Then he takes a decision. But if the collection is a lot of pay-off then hand calculation is very hard and time consuming. This suggests us, in very general terms, of computational procedure of game problems which is the fact of combined computer technique.

\footnotetext{
*Author for correspondence. e-mail: hkdas_math@du.ac.bd
}

Game theory has been widely recognized as an important tool in many fields. Since 1950's a lot of scholars have been done on game theory and is continuing today to improve the existing game theory methods and to develop new techniques or models. This paper deals with such existing techniques and LP model and their modification. Basic of game theory models and real life applications are illustrated in this paper. There are some methods to solve the twoperson zero-sum game but all of those cannot be applied to solve all type of game problems. Each of them is suitable for particular cases. A modification of two person zero sum game method is improved which is the pivotal of this research. We will also develop relevant computer code to combine the existing methods through MATHEMATICA ${ }^{3,7}$.

Rest of the paper is organized as follows. The Section II, briefly discuss on some relevant existing methods for solving game problems. The section III, describes rectangular $2 \times 2$ game briefly. The prime goal on the Section IV describes on the $m \times n$ game, algorithm and Programming code. In Section $\mathrm{V}$ is based on the computational experiments.

\section{Existing Methods of Game Problems}

In this section, we present some existing techniques for solving two person zero sum game problems with their merits and demerits.

\section{Graphical method}

Graphical method may be used whenever one of the two players has only two (undominated) pure strategies. Actually, this method advices us to reduce the given $2 \times n$ or $2 \times \mathrm{m}$ game to a much simpler $2 \times 2$ game since every $2 \times \mathrm{n}$ or $2 \times$ m games contains a $2 \times 2$ sub-game such that the optimal minimax strategies for the $2 \times 2$ sub game are also optimal minimax strategies for the $2 \times \mathrm{n}$ or $2 \times \mathrm{m}$ game, with zero probability assigned to each column not in the $2 \times 2$ subgame.

\section{Algebraic method}

In this method we find the mixed strategies by the system of equation solves. If the system of equations inconsistent, then we conclude that at least one of the inequalities is a strict inequality. This system concludes a number of equations that is very difficult to solve for many variables. 
Method of sub-games

In this method sub-divides the $2 \times n$ or $m \times 2$ game into a number of $2 \times 2$ games. Each of these sub-games is solved by any method and then optimum strategies are selected. This method is limited for $2 \times n$ or $m \times 2$ games. For this reason, in the large game problems it will be failed.

\section{Matrix method}

This method solves $m \times n$ game problems quite efficiently. Firstly, it takes two matrices for the players and then needs to find row oddments and column oddments which costs much more time compared with other methods in case of higher orders. If the sum of row oddments is equal to the sum of the column oddments then we can find the optimal strategies for the players, otherwise the method fails.

\section{Iterative method}

One of the most practical methods for solving those large games which are not reducible to smaller convenient size is Iterative method or Brown's algorithm. Its calculation is not so much tedious and hard. But, it gives an approximate solution for the value of the game. To get the optimal strategies, it assumes that each player acts under the assumption that the past is the best guide to the future.

\section{Dominance method}

This is very important method to reduce the game size of the payoff matrix. If no saddle point is found in a game there is no single safest strategy for each player. In that case, a mixture of strategies is used and this is done by this method on the payoff matrix which can be reduced if it is possible to eliminate certain strategies by dominance. Then resulting reduced game can be solved for finding mixed strategy. Theoretically and practically, it is true to deal with many game problems which are in higher dimensions causing a great trouble for finding the optimal solutions with their strategies.

\section{Rectangular $2 \times 2$ Game}

In this section, we present a brief discussion about $2 \times 2$ rectangular game problems. Let $\mathrm{x}_{\mathrm{i}}$ be the probability for player I, plays row II with $\mathrm{i}=1,2$, and let $\mathrm{y}_{\mathrm{j}}$ be the probability for player II plays column $\mathrm{j}$ with $\mathrm{j}=1,2$. Since

\section{Player II}

$$
\text { Player I } \quad\left[\begin{array}{ll}
p_{11} & p_{12} \\
p_{21} & p_{22}
\end{array}\right] \quad \ldots \quad \ldots \quad \text { (1.a) }
$$

$\sum_{i=1}^{2} x_{i}=1$ and $\sum_{j=1}^{2} y_{j}=1$ so we can write $\mathrm{x}_{2}=1-\mathrm{x}_{1}$ and $\mathrm{y}_{2=} 1$ $-\mathrm{y}_{1}$

Then examine the payoff matrix for a saddle point. If one or more exist, the optimal minimax strategies are pure strategies. They are obtained by playing the row and column a saddle point is in with probability 1 , and the other row and column with probability 0 . The saddle point is necessarily the value of the game. If a saddle point does not exist, then we have to follow the following procedure. The optimal strategy of player I is $\bar{x}=\left(\begin{array}{c}\mathrm{x} * 1 \\ \mathrm{x} * 2\end{array}\right)$ and the optimal strategy of player II is $\bar{y}=\left(\begin{array}{l}y * 1 \\ y * 2\end{array}\right)$, where,

$$
\begin{aligned}
& \mathrm{X}^{*}{ }_{1}=\frac{p_{22}-p_{21}}{p_{11}+p_{22}-p_{12}-p_{21}} \ldots \quad \ldots \quad \ldots \quad \ldots
\end{aligned}
$$

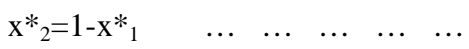

$$
\begin{aligned}
& \mathrm{y}^{*}{ }_{1}=\frac{p_{22}-p_{12}}{p_{11}+p_{22}-p_{12}-p_{21}} \ldots \ldots \ldots
\end{aligned}
$$

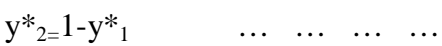

These will be optimal minimax strategies for player I and player II.

Finally the value of the game is

$$
\begin{aligned}
& v=\mathrm{x}_{1} \mathrm{y}^{*}{ }_{1} p_{11}+\mathrm{x}_{1}{ }_{1}\left(1-\mathrm{y}{ }_{1}\right) p_{12}+\left(1-\mathrm{x}_{1}{ }_{1}\right) \mathrm{y}^{*}{ }_{1} p_{21}+\left(1-\mathrm{x}_{1}{ }_{1}\right)(1- \\
& \left.\mathrm{y}_{2}{ }_{2}\right) p_{22} \ldots
\end{aligned}
$$

\section{Formulation of $m \times n$ Game Problems}

In many applications, one needs to compute basic solutions of a system of linear equations. In this paper, we outline a procedure for finding two strategies of a system of $\mathrm{m}$ equations in $n$ variables and develop a computer procedure using the computer language MATHEMATICA. For this, if possible, we first reduce the given pay-off matrix by using the Dominance Method and then applying Minimax-Maxmin method to find Game value. But in case of dominance checking and a modification is mention in here. Actually, we will not completely finish the dominance checking either it may have scope to do this. After that, we convert the dominance result into (2.a) and (2.b) to find their strategies. Finally, we will solve (2.a) and (2.b) by using simplex method.

\section{Discussion of $m \times n$ game}

Any two person zero sum game with mixed strategies can be solved by transforming the problem to a LP. Let, the value of game is $v$. Initially, player I acts as maximize and player II acts as minimize. But after transforming some steps when we convert the LP then inverse the value of the game. For this objective function also changes.

Cosider the optimal mixed strategy for player II, Expected payoff for player II $=\sum_{i=1}^{m} \sum_{j=1}^{n} p_{i j} y_{j} x_{i}$ and the player II strategy $\left(\mathrm{x}_{1}, \mathrm{x}_{2}, \ldots, \mathrm{x}_{\mathrm{m}}\right)$ is optimal if $\sum_{i=1}^{m} \sum_{j=1}^{n} p_{i j} y_{j} x_{i} \leq v$ for all opposing strategies i.e. player $\mathrm{I}$ is $\left(\mathrm{y}_{1,}, \mathrm{y}_{2}, \ldots \ldots . ., \mathrm{y}_{\mathrm{n}}\right)$. Finally, we have the followng forms for the player II and player I respectively.

Player II :

Maximize $\frac{1}{v}=x_{1}+x_{2}+\ldots \ldots \ldots \ldots \ldots \ldots+x_{m}$

Subject to,

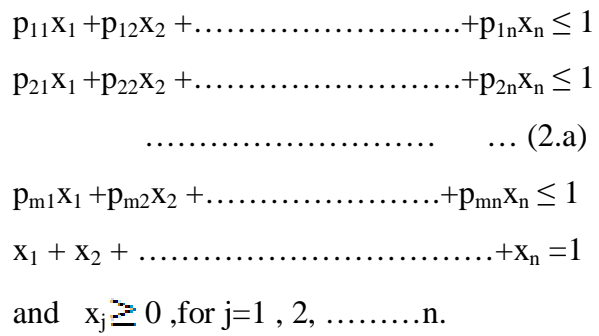


Player I:

Minimize $\frac{1}{v}=\mathrm{y}_{1}+\mathrm{y}_{2}+\ldots \ldots \ldots \ldots \ldots \ldots \ldots+\mathrm{y}_{\mathrm{m}}$

Subject to,

$\mathrm{p}_{11} \mathrm{y}_{1}+\mathrm{p}_{21} \mathrm{y}_{2}+\ldots \ldots \ldots \ldots \ldots \ldots+\mathrm{p}_{\mathrm{m} 1} \mathrm{y}_{\mathrm{m}} \geq 1$

$\mathrm{p}_{12} \mathrm{y}_{1}+\mathrm{p}_{22} \mathrm{y}_{2}+\ldots \ldots \ldots \ldots \ldots \ldots \ldots+\mathrm{p}_{\mathrm{m} 2} \mathrm{y}_{\mathrm{m}} \geq 1$

$\mathrm{P}_{1 \mathrm{n}} \mathrm{y}_{1}+\mathrm{p}_{2 \mathrm{n}} \mathrm{y}_{2}+\ldots \ldots \ldots \ldots \ldots \ldots+\mathrm{p}_{\mathrm{mn}} \mathrm{y}_{\mathrm{m}} \geq 1$

$\mathrm{y}_{1}+\mathrm{y}_{2}+\ldots \ldots \ldots \ldots \ldots \ldots \ldots \ldots \ldots+\mathrm{y}_{\mathrm{m}}=1$

and $\mathrm{y}_{\mathrm{i}} \geq 0$,for $\mathrm{i}=1,2, \ldots \ldots \ldots \ldots \ldots \mathrm{m}$.

We can solve (2.a) and (2.b) by suitable LP method such as usual simplex method or Big M simplex method or Primaldual simplex method ${ }^{7}$. But we will solve (2.a) and (2.b) by using simplex method by using computer code.

Algorithm of $m \times n$ game

In this section, we first propose a combined algorithm which first implement the dominance rule to reduce the game of the linear system of equation $\mathrm{Ax}=\mathrm{b}, \mathrm{x} \geq 0$ for the generalizations $m \times n$ game and is powerful for the large game problems and then we apply rectangular $2 \times 2$ game, MinimaxMaximin and Modified matrix. Finally, to find the strategies of two players we use another algorithm.

Step 1: If the given pay-off matrix is $2 \times 2$ then go to Step 3 otherwise go to step 2.

Step 2: Eliminate all recessive rows and columns of the payoff matrix by the following Sub step.

Sub Step a: Each value in the dominating rows must be greater than or equal to the corresponding value of the dominated rows.

Sub Step b: Delete the dominated rows.

Sub Step c: Each value in the dominating columns must be less than or equal to the corresponding value of the dominated columns.

Sub Step d: Eliminate the dominating columns.

Sub Step e: If all row and column checking complete then go to Step 4.

Step 3: If the reduced pay-off matrix is $2 \times 2$ then find the Game value with their strategies.

Sub Step I: Search the maximum element from each row of the reduced payoff matrix of equation (1.a).

Sub Step II: Search the minimum element from each column of the reduced payoff matrix of equation (1.a).

Sub Step III: If they coincide then the value of the game is $\mathrm{V}=$ Maximin element=Minimax element. Then Stop. When we fail to get such value, go to Sub Step IV.

Sub Step IV: Find the mixed strategies for player I using (1.b) and (1.c).
Sub Step V: Find the mixed strategies for player II using (1.d) and (1.e).

Sub Step VI: Finally, we have to get value of the game by (1.f). Otherwise, go to Step 4.

Step 4: Search the minimum element from each row of the reduced payoff matrix and then find the maximum element of these minimum elements.

Step 5: Search the maximum element from each column of the reduced payoff matrix and then find the minimum element of these maximum elements

Step 6: For the player I, if the Maximin less than zero then find $\mathrm{k}$ which is equal to addition of one and absolute value of Maximin.

Step 7: For the player II if the Minimax less than zero then find $\mathrm{k}$ which is equal to addition of one and absolute value of Minimax.

Step 8: If Maximin and Minimax both are greater than zero then $\mathrm{k} \geq 0$.

Step 9: Finally, to get the modified payoff matrix adding $\mathrm{k}$ with each payoff elements of the given payoff matrix.

Step 10: Then to find the mixed strategies with game value of the two players, follow the following algorithm.

\section{Computer technique of $m \times n$ game}

The Program in this Section is the general program for $m \times n$ game problems corresponding to the above algorithm. This algorithm and computer technique convert the original game problems into reduced matrix (RM) or modified matrix(MM) form. This RM or MM payoff matrix is used in the following Section for finding their strategies with the game value.

\section{Algorithm of Player I and Player II}

In this section, we present our computational procedure incorporated with simplex method in terms of some steps for finding the both players strategy with the game value from the modified matrix.

Step (1): Take the modified payoff matrix for the player II and player I and the value of $\mathrm{k}$.

Step (2): We will get equations (2.a) and (2.b) for the player II and player I respectively.

Step (3): We take input for player II from the equation (2.a).

Step (4): Define the types of constraints. If all are of " $\leq$ " type go to step (6).

Step (5): We follow the following sub-step.

Sub-step (I): Express the problem in standard form.

Sub-step (II): Start with an initial basic feasible solution in canonical form and set up the initial table.

Sub-step (III): Use the inner product rule to find the relative profit factors $\overline{\boldsymbol{c}_{\boldsymbol{J}}}$ as follows $\overline{\boldsymbol{c}_{\boldsymbol{J}}}=\boldsymbol{c}_{\boldsymbol{j}}-\boldsymbol{z}_{\boldsymbol{j}}=\boldsymbol{c}_{\overline{\boldsymbol{J}}}$ - (inner product 
of $\boldsymbol{c}_{\boldsymbol{B}}$ and the column corresponding to $\boldsymbol{x}_{\boldsymbol{j}}$ in the canonical system).

Sub-step (IV): If all $\overline{\boldsymbol{c}_{\boldsymbol{J}}} \leq \mathbf{0}$, the current basic feasible solution is optimal and stop. Otherwise select the non-basic variable with most positive $\overline{\boldsymbol{c}_{\boldsymbol{J}}}$ to enter the basis.

Sub-step $(V)$ : Choose the pivot operation to get the table and basic feasible solution.

Sub-step (VI): Go to Sub-step (III).

Step (6): At first express the problem in standard form by introducing slack and surplus variables. Then express the problem in canonical form by introducing artificial variables if necessary and form the initial basic feasible solution. Go to Sub-step (III).

Step (7): If any $\overline{\boldsymbol{c}_{\boldsymbol{J}}}$ corresponding to non-basic variable is zero, the problem has alternative solution, take this column and go to Sub-step (V).

Step (8): Finally, we find all the stratigies for player II is in corresponding their right hand side (RHS) and strategies of player $\mathrm{I}$ is in corresponding the $\overline{\boldsymbol{c}_{\boldsymbol{j}}}=\boldsymbol{c}_{\boldsymbol{j}}-\boldsymbol{z}_{\boldsymbol{j}}$ of the slack variables.

Step (9): Calculate the value of the object functions for each feasible solution.

\section{Computer technique of $m \times n$ game}

The Program in this Section is the general computer technique which is used to find two players strategy with the game value from the modified matrix for $m \times n$ game problems. The developed computer technique is not presented here for the page limitations.

\section{Computational Experiments}

The efficiency of our technique claimed from the very beginning in this paper is exhibited in the following through numerical experiment.

\section{Test Problems}

The problems are presented here draws upon mainly from two sources, namely the papers H. K. Das, Saha and Hasan" H. K. Das and Hasan ${ }^{6}$, which illustrate the implementation and advantage of the current procedure.

\section{Numerical Example 1}

Two oil companies, USA Oil Co. and Caltex, operating in a city, are trying to increase their market at the expense of the other. The USA Oil Co. is considering possibilities of decreasing price, giving free soft drinks on Rs. 40 purchases of oil or giving away a drinking glass with each 40 litter purchase. Obviously, Caltex cannot ignore this and comes out with its own program to increase its share in the market. The payoff matrix forms the viewpoints of increasing or decreasing market shares is given in table below.
Table 1. $3 \times 3$ payoff matrix games

\begin{tabular}{|c|c|c|c|}
\hline $\mathrm{USA}_{\mathrm{USA}} \mathrm{Cal}$. & I & II & III \\
\hline 1 & $4 \%$ & $1 \%$ & $-3 \%$ \\
\hline 2 & 3 & 1 & 6 \\
\hline 3 & -3 & 4 & -2 \\
\hline
\end{tabular}

Determine the optimum strategies for the oil companies.

Solution: Best strategies for A (Bangladesh Oil Co. $)=(1 / 4$, $1 / 2,1 / 4)$, Best strategies for B (Caltex) $=(27 / 92,62 / 92$, $3 / 92$ ), and Value of the game (for $\mathrm{A}$ ) $=7 / 4$.

\section{Numerical Example 2}

Two candidates in an election campaign selected three cities as crucial and potentially worth a last visit. A survey report indicates the estimated gain ( in thousand votes) for candidate $\mathrm{A}$ as follows. Find the priorities of cities to be visited by both the candidates. Satisfy that the value is $1 / 3$ for either of the candidates.

Table 2. $3 \times 3$ payoff matrix games

\begin{tabular}{|c|c|c|c|}
\hline Can. A & I & II & III \\
\hline 1 & 12 & -9 & 14 \\
\hline 2 & -8 & 7 & 12 \\
\hline 3 & 11 & -10 & 10 \\
\hline
\end{tabular}

Solution: Best strategies for candidates $\mathrm{A}=(5 / 12,7 / 12,0)$, Best strategies for candidates $B=(4 / 9,5 / 9,0)$ and the game value $1 / 3$.

Numerical Example 3

The payoff matrix of a game is given below.

Table 3. $4 \times 5$ payoff matrix games

\begin{tabular}{|l|l|l|l|l|l|}
\hline \multicolumn{1}{|c|}{ Player B } & I & II & III & IV & V \\
Player A & & & & & \\
\hline I & 9 & 3 & 1 & 8 & 0 \\
\hline II & 6 & 5 & 4 & 6 & 7 \\
\hline III & 2 & 4 & 3 & 3 & 8 \\
\hline IV & 5 & 6 & 2 & 2 & 1 \\
\hline
\end{tabular}

Find the best strategy for each player, and the value of a play of the game to $\mathrm{A}$ and $\mathrm{B}$.

Solution: The value of the game is 4 . The strategy for player A is $[0,1,0,0]$. Also the strategy for player $\mathrm{B}$ is $[0,0,1,0,0]$.

\section{Numerical Example 4}

Find the game value and the strategies the following game problems.

Table 4. $4 \times 4$ payoff matrix games

\begin{tabular}{|l|l|l|l|l|}
\hline B A & I & II & III & IV \\
\hline 1 & & & & \\
\hline 2 & 3 & 2 & 4 & 0 \\
\hline 3 & 3 & 4 & 2 & 4 \\
\hline 4 & 4 & 2 & 4 & 0 \\
\hline
\end{tabular}


Solving this game problem by the method of LP, we observe that, both the players strategies does not work properly. So we don't predict which payer will be winning. But if we solve this problem by our combined propose algorithm then it will be solved without any haphazard situation.

Solution: The game value is $8 / 3$, Player A strategies is $(0,0$, $2 / 3,1 / 3)$ and Player $\mathrm{B}$ is $(0,0,2 / 3,1 / 3)$.

\section{Numerical Example 5}

Dhaka University (DU) and Glasgow University (GU) are setting up their strategies for the 2011 international championship university basketball game. Assessing, the strengths of their respective "benches" each coach camp up with four strategies for rotating their players during the game. The ability of each team in scoring 2 pointers, 3 pointers, and free throws is a key factor in determining the final score of the game. The following table summarizes the net points GU will score per possession as a function of the different strategies contemplated by each team:

Table 5. $4 \times 4$ payoff matrix games

\begin{tabular}{|l|c|c|c|c|}
\hline $\mathrm{D} \mathrm{U}$ & $G U_{1}$ & $G U_{2}$ & $G U_{3}$ & $G U_{4}$ \\
\hline$D U_{1}$ & & & & \\
\hline$D U_{2}$ & 3 & -2 & 1 & 2 \\
\hline$D U_{3}$ & 2 & 3 & -3 & 0 \\
\hline$D U_{4}$ & -1 & 2 & -2 & 2 \\
\hline
\end{tabular}

Determine the strategy for the championship game and which of the teams is projected to win the championship?

Solution: The game value is .5, Player DU strategies are $(0$, $0.5,0, .5)$ and Player GU is $(0.14,0.34,0.27,0.25)$.

\section{Numerical Example 6}

Consider the game with the following pay-off matrix. Find the game value with their strategies.

Table 6. $3 \times 4$ payoff matrix games

\begin{tabular}{|c|c|c|c|c|}
\hline$B \quad A$ & I & II & III & IV \\
\hline 1 & 3 & 6 & 1 & 4 \\
\hline 2 & 5 & 2 & 4 & 2 \\
\hline 3 & 1 & 4 & 3 & 5 \\
\hline
\end{tabular}

Solution: The game value is $13 / 4$ and player A strategies is $(1 / 8,1 / 2,3 / 8)$ and Player GU is $(1 / 12,5 / 12,1 / 2,0)$.

\section{Numerical Example 7}

Find the game value and the strategies the following game problems.

Table 7. $4 \times 5$ payoff matrix game

\begin{tabular}{|l|l|l|l|l|l|}
\hline B A & I & II & III & IV & V \\
\hline 1 & 1 & 3 & 2 & 7 & 4 \\
\hline 2 & 3 & 4 & 1 & 5 & 6 \\
\hline 3 & 6 & 5 & 7 & 6 & 5 \\
\hline 4 & 2 & 0 & 6 & 3 & 1 \\
\hline
\end{tabular}

Solution: The game value is 5 and player A strategies is $(0,0,1,0,0)$ with Player B is $(0,1,0,0,0)$.

\section{Numerical Example 8}

Consider the game with the following pay-off matrix.

Table $8.3 \times 3$ payoff matrix game

\begin{tabular}{|l|l|l|l|}
\hline B & I & II & III \\
\hline 1 & & & \\
\hline 2 & 3 & -4 & 2 \\
\hline 3 & 1 & -3 & -7 \\
\hline
\end{tabular}

Solve the above game problem with their strategies also.

Solution: The game value is $4 / 13$ and player A strategies is $(6 / 13,0,7 / 13)$ with Player B is $(8 / 13,5 / 13,0)$.

\section{Numerical Example 9}

Consider the following pay-off matrix.

Table 9. $3 \times 3$ payoff matrix game

\begin{tabular}{|l|l|l|l|}
\hline B & I & II & III \\
\hline 1 & 1 & -2 & 3 \\
\hline 2 & 1 & 3 & -2 \\
\hline 3 & 4 & 2 & 1 \\
\hline
\end{tabular}

Solve the above game problem with their strategies.

Solution: The game value is 1.33 and player A strategies is $(1 / 6,0,5 / 6)$ with Player B is $(0,1 / 3,2 / 3)$.

\section{Numerical Example 10}

Three homeowners - a carpenter, an electrician and a plumber - agree to make repairs in their three homes. They agree to work a total of 10 days each according to the following schedule.

Table $10.3 \times 3$ payoff matrix game

\begin{tabular}{|l|l|l|l|}
\hline \multicolumn{1}{|c|}{ Perf. Work } & Carp. & Elec. & Plum. \\
Days of work & & & \\
\hline Carpenter & 2 & 1 & 6 \\
\hline Electrician & 4 & 5 & 1 \\
\hline Plumber & 4 & 4 & 3 \\
\hline
\end{tabular}

Determine the optimum strategies for the owners.

\section{Method comparison}

We introduce here that some existing method may fails but our single framework is successful in those situations. Let, $\mathrm{S}$ stands for Success and F stands for Fail. In the following table, we see that there are some methods to solve the twoperson zero-sum game but not all of those methods can be applied to solve all type of problems. Each of them is suitable for particular cases. In briefly, example 8 also fails for the Matrix method. 
Table 11. Accuracy of the algorithm

\begin{tabular}{|c|c|c|c|c|c|c|c|c|}
\hline \multirow[t]{2}{*}{ No: } & \multicolumn{5}{|c|}{ All Established method } & \multirow[b]{2}{*}{ Ref. 5} & \multirow[b]{2}{*}{ Ref. 6} & \multirow{2}{*}{$\begin{array}{l}\text { Current } \\
\text { algorithm }\end{array}$} \\
\hline & Dominance & L P & Graphical & $\begin{array}{l}\text { Minimax } \\
\text { Maximin }\end{array}$ & Iterative & & & \\
\hline 1 & $\mathrm{~F}$ & $\mathrm{~S}$ & $\mathrm{~F}$ & $\mathrm{~F}$ & less accuracy & $\mathrm{S}$ & $\mathrm{S}$ & $\mathrm{S}$ \\
\hline 2 & $S$ & $S$ & $\mathrm{~F}$ & $\mathrm{~F}$ & less accuracy & $S$ & S & $\mathrm{S}$ \\
\hline 3 & $S$ & $S$ & $\mathrm{~F}$ & $S$ & less accuracy & $S$ & $\mathrm{~S}$ & $\mathrm{~S}$ \\
\hline 4 & S & $\mathrm{F}$ & $\mathrm{F}$ & $\mathrm{F}$ & less accuracy & $S$ & $\mathrm{~F}$ & $\mathrm{~S}$ \\
\hline 5 & $\mathrm{~S}$ & $\mathrm{~S}$ & $\mathrm{~F}$ & $\mathrm{~F}$ & less accuracy & $S$ & $\mathrm{~S}$ & $\mathrm{~S}$ \\
\hline 6 & $\mathrm{~F}$ & $S$ & $\mathrm{~F}$ & $\mathrm{~F}$ & less accuracy & $\mathrm{S}$ & $\mathrm{S}$ & $S$ \\
\hline 7 & $S$ & $\mathrm{~F}$ & $\mathrm{~F}$ & $\mathrm{~S}$ & less accuracy & $S$ & $\mathrm{~F}$ & $S$ \\
\hline 8 & $\mathrm{~F}$ & $\mathrm{~S}$ & $\mathrm{~F}$ & $\mathrm{~F}$ & less accuracy & $S$ & $\mathrm{~S}$ & $S$ \\
\hline 9 & $\mathrm{~F}$ & $S$ & $\mathrm{~F}$ & $\mathrm{~F}$ & less accuracy & $S$ & $S$ & $S$ \\
\hline 10 & $\mathrm{~F}$ & $\mathrm{~S}$ & $\mathrm{~F}$ & $\mathrm{~F}$ & less accuracy & $\mathrm{S}$ & $\mathrm{S}$ & $\mathrm{S}$ \\
\hline
\end{tabular}

\section{Coding comparison}

We give a time comparison chart for the efficiencies of our combined computer technique with the some developed computer techniques. Also, we use the computer configuration as: Processor: Intel(R) Pentium(R) Dual CPU
E2180@2.00GHZ 2.00GHZ, Memory (RAM):1.00 GB and the System type: 32-bit operating system. To find the run time of our implementation code we use "TimeUsed[ ]" command and we define time consuming by TC.

Table 12. Accuracy of the computer techniques

\begin{tabular}{|l|l|l|}
\hline Character & Reference 5 and Our Program & Brown's Program \\
\hline Numerical Example & $\begin{array}{l}\text { Reference problem No. 5.1.9 and current example } \\
\text { No. } 9\end{array}$ & Reference problem No. 5.1.9 \\
\hline Computer information & $\begin{array}{l}\text { Processor: Intel(R) Pentium(R) Dual CPU } \\
\text { E2180@ 2.00GHZ 2.00GHZ, Memory (RAM):1.00 }\end{array}$ & $\begin{array}{l}\text { 48 K bytes core storage. } \\
\text { IBM 370/168 computer }\end{array}$ \\
\cline { 2 - 3 } & GB and the System type: 32-bit operating system. & \\
\hline Iteration Number & Third & 500 \\
\hline Result & Exact & Approximation \\
\hline Time & 0.272 seconds & 0.51 seconds \\
\hline
\end{tabular}

Table 13. Accuracy of the computer techniques

\begin{tabular}{|c|c|c|c|c|}
\hline Number & $\begin{array}{c}\text { Manual } \\
\text { Calculation }\end{array}$ & $\begin{array}{c}\text { Total coding } \\
\text { time in reference 5 }\end{array}$ & $\begin{array}{c}\text { Total coding } \\
\text { time in reference 6 }\end{array}$ & $\begin{array}{c}\text { Total coding time in the } \\
\text { current computer technique }\end{array}$ \\
\hline 1 & TC & 0.376 & 0.276 & 0.126 \\
\hline 2 & TC & 0.134 & 0.123 & 0.101 \\
\hline 3 & TC & 0.483 & 0.383 & 0.1253 \\
\hline 4 & TC & 0.389 & F & 0.124 \\
\hline 5 & TC & 0.354 & 0.234 & 0.126 \\
\hline 6 & TC & 0.297 & 0.287 & 0.145 \\
\hline 7 & TC & 0.464 & F & 0.101 \\
\hline 8 & TC & 0.346 & 0.235 & 0.121 \\
\hline 9 & TC & 0.272 & 0.054 & 033 \\
\hline
\end{tabular}

\section{Conclusion}

This paper improved a game theoretic algorithm and developed its computer-oriented program for solving two person zero sum game problems. A comparative study had presented here and discussed the changes step by step. The program developed here is a powerful computer technique. A number of numerical examples illustrated in this paper to demonstrate the developed computer technique. 


\section{References}

1. Bansal, B. M.C. Puri, 1980. A Min Max Problem, Zeitschrift fur operations Research, 24, 191-200.

2. Brown K. L., Shoam, 2009. Multigent Systems algorithmic, Game Theoric, and Logical Foundations, Cambridge University Press.

3. Dantzig G. B., 1962. Linear Programming and extension, Princeton university press,Princeton,N,J.

4. Davis M., 1983. Game Theory: An Introduction, New York.

5. Das, H.K., T. Saha and M.B. Hasan, 2012. Numerical Experiments By Improving a Numerical Methods for Solving Game Problems Through Computer Algebra, Int. Journal of Decision Sciences, 3(1), 23-52.

6. Das, H.K. and M.B. Hasan, 2011. An Algorithm and Its Computer Technique for Solving Game Problems Using LP method, Int. J. of Basic \& Applied Sciences", 11(3), 90-99.

7. Don Eugene., 2001. Theory and Problems of Mathmatica, Schaum's Outline Series, McGraw-Hill, New york San Francisco Washington, D.C.

8. Hofbauer J., Oeachsslen J. and Riedel F., 2009. Brown-von Neumann -Nash Dynamics: The continuous strategy case, Games and Economic Behaviour, 65(2), 46-429.

9. Karak P.M., 2001. Linear Programming and Theory of Games, Chhaya Prakashani, Calcutta.
10. Mayer B., Yoella and A. E. Roth, 2006. Learning in Nosy Games and the Sustainability of Cooperation, American Economic Review, 96(4), 1029-1042.

11. Meyerson, R. B., 1991. Game Theory: Analysis of Conflict, Harvard University Press, Cambridge.

12. Nisan N., Roughgarden T., E. Tardos, V.V. Vazirari, 2007. Algorithmic Game Theory, Cambridge university press.

13. Von Neumann J., and O. Morgenstern, 1947. Theory of Games and Economic Behavior", second edition, Princeton University Press, Princeton, N.J.

14. Philip D. Straffin, 1993. Game Theory and Strategy, Mathematical Association of America.

15. Guillermo Owen, 1982, Game Theory by, 2nd edition, Academic Press.

16. Das, H.K., 2016. Investigation on the Theory of Games and Its Economic Impact, UGC Research Project, Dhaka University, Dhaka-1000, 2015- 2016.

17. Das H.K. and Sajal Chakroborty, 2016. Analyzing on the Decomposition based Pricing Procedure for solving two Person Zero Sum Game Problems through Computer Algebra, International Journal of Computer Applications, 156(12), 37-47. 
H. K. Das 